\title{
The appropriateness of emergency medical service responses in the eThekwini district of KwaZulu-Natal, South Africa
}

\author{
P R Newton, ${ }^{1}$ MTech (Emergency Medical Care); R Naidoo, ${ }^{1}$ MSc (Cardiology); P Brysiewicz, ${ }^{2}$ PhD (Health Science) \\ ${ }^{1}$ Department of Emergency Medical Care and Rescue, Faculty of Health Sciences, Durban University of Technology, South Africa \\ ${ }^{2}$ School of Nursing and Public Health, College of Health Sciences, Nelson R Mandela School of Medicine, University of KwaZulu-Natal, \\ Durban, South Africa
}

Corresponding author: P R Newton (p.newton@brookes.ac.uk)

\begin{abstract}
Introduction. Emergency medical services (EMS) are sometimes required to respond to cases that are later found not to be emergencies, resulting in high levels of inappropriate responses. This study evaluated the extent to which this occurs.

Methods. All cases dispatched over 72 hours by the eThekwini EMS in Durban, South Africa, were prospectively enrolled in a quantitative descriptive study. Vehicle control forms containing dispatch data were matched and compared with patient report forms containing epidemiological and clinical data to describe the nature and extent of inappropriate responses based on patient need. Data were subjected to simple descriptive analysis, correlations and $\chi^{2}$ testing.

Results. A total of 1385 cases met the study inclusion criteria. Marked variations existed between dispatch and on-scene priority settings, most notably in the highest priority 'red-code' category, which constituted $>56 \%$ of cases dispatched yet accounted for $<2 \%$ at the scene $(p<0.001)$. Conversely, $>80 \%$ of 'red-code' dispatches required a lower priority response. When comparing resource allocation according to patient interventional needs, $>58 \%$ of cases required either no intervention or transport only and almost $36 \%$ required basic life support intervention only $(p<0.001)$. Moreover, $<12 \%$ of advanced life support dispatches were for patients found to be 'red code' at the scene.

Conclusion. There is a significant mismatch between the dispatch of EMS resources and actual patient need in the eThekwini district, with significantly high levels of inappropriate emergency responses.
\end{abstract}

S Afr Med J 2015;105(10):844-847. DOI:10.7196/SAMJnew.8273 


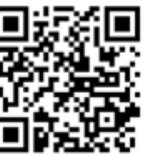

Emergency medical services (EMS) attend cases involving patients who have been classified as having time-critical life-threatening illnesses or injuries. An EMS unit will typically respond to these cases on the basis of information provided by a caller to the EMS provider's emergency medical communications centre (EMCC). Before the unit is dispatched, the caller, who may or may not be the patient, is routinely questioned by an emergency medical dispatcher, who ascertains the level of EMS response required based on the answers provided. When the responding EMS unit arrives at the scene, however, it is frequently found that many of these patients do not in fact represent an emergency; high levels of inappropriate EMS responses may therefore be occurring. Furthermore, many of the patients who are transported do not have a clearly defined need for EMS intervention, ${ }^{[1]}$ yet there is an expectation that they must be taken to a medical facility. When these mismatches occur, the response may be deemed to be inappropriate.

A response may be inappropriate in several ways, ${ }^{[2]}$ i.e. when an EMS provider:

- commits an underutilisation of resources for critical patients, resulting in an unmet need

- commits an overutilisation of resources for persons with needs not ideally addressed by higher levels of EMS care

- commits an overutilisation of resources for persons with needs not ideally addressed by EMS at all, and/or

- commits resources to a response utilising 'emergency response procedures' to non-time-sensitive acute illness or injury.

The reasons for inappropriate responses are not fully understood and are thought to be complex and varied. Reasons cited include public ignorance or misunderstanding relating to the use of EMS systems, poor or unavailable alternative public transport, inaccessible or distant primary healthcare facilities, deliberate misrepresentation or exaggeration of need, and malicious hoax calls. ${ }^{[3]}$

Whether responses to calls are appropriate or inappropriate, the potential for vehicle or pedestrian collisions is often present. The unnecessary use of emergency driving procedures with lights and sirens may expose responding EMS crews and members of the public to an increased risk of serious injury or even death. ${ }^{[4]}$ Moreover, once the responding unit arrives at the scene, the EMS crew is compelled to transport the patient to hospital, irrespective of his or her condition. This results in the unit being tied up in a system with already limited resources, frequently leaving no more units available to respond to further incoming calls.

Studies conducted in developed countries suggest that widespread inappropriate use of EMS systems has been evident since the 1970s and that up to $52 \%$ of all requests for an EMS response are later found to be inappropriate. ${ }^{[5]}$ Three studies have been undertaken in South Africa (SA). Frank and De Villiers ${ }^{[6]}$ showed that up to $68 \%$ of the cases transported by the Caledon EMS in Overberg, Western Cape Province, were not emergencies and that the inappropriate use of EMS units resulted in unnecessary costs. McFarlane et al. ${ }^{[7]}$ highlighted high levels of inappropriate use of EMS vehicles in the greater Johannesburg area, which overwhelmed a system that was already severely under-resourced, and Meents and Boyles ${ }^{[8]}$ showed that $16.7 \%$ of all EMS requests in the Eastern Cape Province were never dispatched.

In KwaZulu-Natal Province (KZN) there have been no studies relating to the possible inappropriate use of EMS resources, and there is a paucity of evidence relating to EMS in general. The studies mentioned in the preceding paragraph cannot be generalised to either KZN or the eThekwini district owing to differences in demographics, disease profiles, geography, and EMS operating systems and procedures.

\section{Objective}

To evaluate the appropriateness of EMS responses in the eThekwini district of KZN by asking whether the various levels of response assigned on dispatch adequately and routinely met the clinical needs of patients in terms of resource allocation.

\section{Methods}

\section{Research setting and population}

The study was conducted in the eThekwini health district of KZN, one of the province's 11 health districts, encompassing the greater Durban area. The population of eThekwini was last recorded in the 2007 community census at almost 3.5 million people and the population density is estimated at 1394 people per $\mathrm{km}^{2}$, with approximately $10 \%$ living in underdeveloped informal settlements.

The study population comprised all EMS responses undertaken by the KZN Department of Health within the eThekwini health district, which according to unpublished statistical data averaged in excess of 400 daily.

\section{Sample}

Purposeful sampling comprised emergency calls to the eThekwini health district EMCC placed, logged and physically responded to over a busy 72-hour month-end period, 24 hours of which included a weekend peak period. By spreading the sampling over this period, the data collected gave a fair representation of the different categories of cases responded to and of the rates and frequencies of these responses.

Data were collected over the period $24-26$ November 2011, the first available period as described above immediately following ethical and organisational approval. Altogether 1689 responses were recorded, of which 1385 met the study inclusion criteria. A total of 304 records were excluded because data were incomplete or a dispatch did not actually occur (e.g. cancelled or duplicated cases).

\section{Data collection and analysis}

Computerised dispatch logs were accessed through the EMCC's Zetron DCS-5020 digital console system, from which vehicle control forms were generated. Data extracted comprised the dispatch priority setting and the initial level of resource allocation. These were then matched and compared with the corresponding EMS unit patient report form, from which data relating to the on-scene priority setting and the required level of patient interventions were extracted.

Dispatch and on-scene priority settings were categorised using a standardised triage scale, and the resource allocation and patient interventions were categorised against the capabilities of the responding emergency care providers as defined by the Health Professions Council of South Africa. ${ }^{[9]}$

Data were then entered into IBM Statistics SPSS version 21 and, in consultation with a statistician, subjected to simple descriptive analysis, correlations and $\chi^{2}$ testing.

\section{Ethical considerations}

Ethical approval was granted by the Durban University of Technology's Institutional Research Ethics Committee (ref. FHSEC 009/11) and the KZN Department of Health Research and Knowledge Management sub-component (ref. HRKM 159/11).

\section{Results}

There were more female than male patients $(n=699$ (50.5\%) and $n=645$ (46.6\%), respectively). The gender of the patient could not 
be established in 41 cases (2.9\%). Patients ranged in age from newborn to 99 years old (mean 32.5 years for males and 33.1 years for females).

Marked variations existed between dispatch and on-scene priority settings, which were most notable in the highestpriority, life-threatening 'red-code' category. This category constituted $>56 \%$ of cases dispatched yet accounted for $<2 \%$ at the scene $(p<0.001)$. These figures represent an overtriage rate of $>93 \%$ (Fig. 1). Similar albeit less notable results were seen in the yellow-code (urgent but non-life-threatening) category, with an over-triage rate of just over $13 \%$ and an under-triage rate of $<2 \%$. The green-code (non-urgent) category, however, showed a disturbing under-triage rate of $>82 \%$, with almost 1 in 10 patients being found to be red code by responding crews once at the scene (Table 1).

When comparing resource allocation according to patient interventions, $>58 \%$ of cases attended required no intervention or a means of transport only and $<36 \%$ required basic life support (BLS) intervention $(p<0.001)$. Patients requiring intermediate life support (ILS) and advanced life support (ALS) interventions comprised $4.0 \%$ and $1.4 \%$, respectively. When BLS resources were dispatched, $<2 \%$ of patients required intervention at a higher level. Conversely, when ALS resources were dispatched, almost $61 \%$ of patients required either no intervention or a means of transport only, with $<15 \%$ actually requiring an ALS intervention (Table 2). Moreover, $<12 \%$ of ALS dispatches were for patients found to be red code at the scene, with $>80 \%$ of dispatches being for patients who were coded either yellow or green at the scene (Table 3).

Patients were transported and delivered to a health institution in a total of 970 (70.0\%) of the 1385 cases in which EMS were dispatched. Patients who were not transported, irrespective of whether they received any intervention or not, totalled 415 (30.0\%). This figure included 271 patients who were never located, leaving the total number of EMS patient contacts at 1121 .

\section{Discussion}

The disagreement rates demonstrated by the high levels of over-triage across the red- and yellow-code categories in this study compare unfavourably with modest rates as low as 26\% reported elsewhere. ${ }^{[10]}$ Under-triage rates, particularly in the green-code category, also compared unfavourably with rates of between $4 \%$ and $7 \%$ reported elsewhere. ${ }^{[11]}$
Similar disagreements were evident when comparing the interventional requirements at the scene with the levels of dispatch. In the majority of cases in which ALS resources were dispatched, patients required no intervention or a means of transport only, leaving less than 2 in 10 patients actually requiring an ALS intervention. Although this mismatch has been evident in EMS systems elsewhere, such high levels of inappropriate ALS dispatch to lower-priority patients have not been seen. Surprisingly, $>95 \%$ of patients required nothing more than a BLS-level intervention.

It is clear from the results presented that appropriate allocation of resources aimed at meeting patient needs is not being achieved and that needs cannot be met effectively owing to the high levels of inconsistencies reported. Of particular concern is the high proportion of cases in which EMS resources are routinely dispatched to cases that either require no intervention or a means of transport only. Such cases represent almost 6 out of 10 dispatches by the eThekwini EMS. This figure is similar to that in one of the studies conducted in SA, ${ }^{[6]}$ with international studies reporting rates of between $11 \%$ and $52 \%{ }^{[5]}$

There is undoubtedly an urgent need for

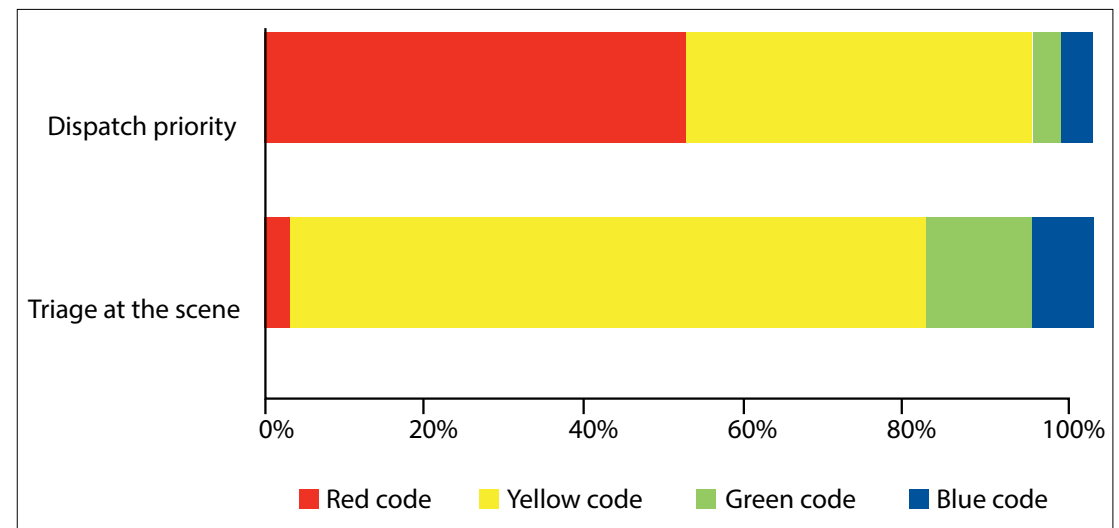

Fig. 1. Comparison of dispatch priority v. triage code at the scene.

Table 1. Comparison of priority/triage allocations between dispatch and at the scene, $n(\%)$

\begin{tabular}{|c|c|c|c|c|c|}
\hline & \multicolumn{4}{|c|}{ Triage code at the scene } & \multirow[b]{2}{*}{ Total } \\
\hline & $\begin{array}{l}\text { Red code/ } \\
\text { priority } 1\end{array}$ & $\begin{array}{l}\text { Yellow code/ } \\
\text { priority } 2\end{array}$ & $\begin{array}{l}\text { Green code/ } \\
\text { priority } 3\end{array}$ & $\begin{array}{l}\text { Blue code/ } \\
\text { priority } 4\end{array}$ & \\
\hline \multicolumn{6}{|l|}{$\begin{array}{l}\text { Triage code on } \\
\text { dispatch }\end{array}$} \\
\hline $\begin{array}{l}\text { Red code/ } \\
\text { priority } 1\end{array}$ & $14(2.4)$ & $469(79.8)$ & $68(11.6)$ & $37(6.3)$ & $588(100.0)$ \\
\hline $\begin{array}{l}\text { Yellow code/ } \\
\text { priority } 2\end{array}$ & $7(1.4)$ & 407 (82.6) & $75(15.2)$ & $4(0.8)$ & $493(100.0)$ \\
\hline $\begin{array}{l}\text { Green code/ } \\
\text { priority } 3\end{array}$ & $3(7.5)$ & $30(75)$ & $7(17.5)$ & $0(0.0)$ & $40(100.0)$ \\
\hline Total & $24(2.1)$ & $906(80.8)$ & $150(13.4)$ & $41(3.7)$ & $1121(100.0)$ \\
\hline
\end{tabular}


Table 2. Qualification level of emergency care provider v. intervention at the scene, $\boldsymbol{n}(\%)$

\begin{tabular}{lllllll}
\hline \multicolumn{5}{c}{} & \multicolumn{5}{c}{ Nature of intervention } & \\
\cline { 2 - 5 } & $\begin{array}{l}\text { None } \\
\text { required }\end{array}$ & $\begin{array}{l}\text { Transport } \\
\text { only }\end{array}$ & BLS & ILS & ALS & Total \\
\hline $\begin{array}{l}\text { Emergency } \\
\text { care provider } \\
\text { qualification }\end{array}$ & & & & & & \\
$\quad$ BLS & $178(25.4)$ & $216(30.8)$ & $294(41.9)$ & $9(1.3)$ & $4(0.6)$ & $701(100.0)$ \\
ILS & $164(26.7)$ & $213(34.6)$ & $193(31.4)$ & $39(6.3)$ & $6(1.0)$ & $615(100.0)$ \\
ALS & $39(56.5)$ & $3(4.3)$ & $9(13.0)$ & $8(11.6)$ & $10(14.5)$ & $69(100.0)$ \\
Total & $381(27.5)$ & $432(31.2)$ & $496(35.8)$ & $56(4.0)$ & $20(1.4)$ & $1385(100.0)$
\end{tabular}

Table 3. Qualification level of emergency care provider v. triage code at the scene, $n$ (\%)

\begin{tabular}{llllll}
\hline & \multicolumn{5}{c}{ Triage code at the scene } \\
\cline { 2 - 5 } & $\begin{array}{l}\text { Red code/ } \\
\text { priority 1 }\end{array}$ & $\begin{array}{l}\text { Yellow code/ } \\
\text { priority 2 }\end{array}$ & $\begin{array}{l}\text { Green code/ } \\
\text { priority 3 }\end{array}$ & $\begin{array}{l}\text { Blue code/ } \\
\text { priority 4 }\end{array}$ & Total \\
\hline $\begin{array}{l}\text { Emergency } \\
\text { care provider } \\
\text { qualification }\end{array}$ & & & & & \\
$\quad$ BLS & $9(1.6)$ & $480(85.9)$ & $61(10.9)$ & $9(1.6)$ & $559(100.0)$ \\
ILS & $10(1.9)$ & $396(76.3)$ & $84(16.2)$ & $29(5.6)$ & $519(100.0)$ \\
ALS & $5(11.6)$ & $30(69.8)$ & $5(11.6)$ & $3(7.0)$ & $43(100.0)$ \\
Total & $24(2.1)$ & $906(80.8)$ & $150(13.4)$ & $41(3.7)$ & $1121(100.0)$ \\
& & & & &
\end{tabular}

where priority dispatch systems are used, and compliance and quality assurance controls are in place, the system is more effective at triaging calls correctly than an emergency medical dispatcher. ${ }^{[14]}$

The eThekwini EMS currently does not make use of a recognised MPDS, and the system in use has few procedures in place to ensure the thorough and structured interrogation of callers. Additionally, only three levels of dispatch priorities are routinely allocated and callers are offered little in terms of pre-arrival instructions. It is important to note that this system is not limited to the eThekwini district but is in widespread use throughout KZN.

\section{Alternative routing of patients}

The need to match patient need to limited EMS resources more efficiently has driven many EMS systems to consider safe alternatives to traditional response methods. At the point of dispatch, these may include referral to non-emergency access numbers, such as the NHS 111 number in the UK, or alternative appropriate services or facilities. $^{[12]}$ Additionally, if patients can be identified as suffering from minor illness or injury once an EMS unit reaches the scene, it may be possible to offer alternatives to emergency ambulance transportation. ${ }^{[13]}$ These alternatives, based on patient presentation, may include a straightforward refusal to transport, treatment at the scene and/or referral to a further care facility by other transportation means, or on-scene treatment and discharge. However, caution should be advised, as few rigorous trials have been reported and the evidence of the safety and efficacy of alternative responses is conflicting and weak.

At present, unless the patient specifically refuses to be transported, the eThekwini EMS transports all patients to hospital irrespective of their condition. Patients with relatively minor illness or injury are frequently transported over extended distances, often bypassing facilities that may be more appropriate. This is also common practice throughout $\mathrm{KZN}$, and arguably wastes expensive and limited resources that could be utilised better elsewhere. Moreover, patients transported directly to hospital may overburden already under-resourced emergency departments.

\section{Study limitations}

There were three main limitations to the study: the cross-sectional design did not take into account seasonal changes in patient need; the sample size was relatively small considering the population; and the population was restricted to the urban and periurban districts of eThekwini. The results might have been slightly different had the study been conducted longitudinally, over an extended period of time, and within the greater SA geographical context.

\section{Conclusions}

The study findings clearly confirm that there is a significant mismatch between the dispatch of EMS resources and actual patient need in the eThekwini district of KZN, with significantly high levels of inappropriate emergency responses. This situation is likely to continue unless strategies designed to optimise the use of limited resources are employed. Further research and development in the areas of medical priority dispatch systems and alternative routing of patients in a wider SA context are needed and encouraged.

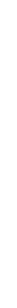

References

1. Gratton MC, Ellison SR, Hunt J, Ma OJ. Prospective determination of medical necessity for ambulance transpor by paramedics. Prehosp Emerg Care 2003;7(4):466-469 [http:// x.doi.org/10.1080/31270300220X]

2. Berry M, Blocker J, Bryant K, et al. A Strategic-based Emergency Medical Services Blueprint for Oklahoma City. Oklahoma City City Council of the City of Oklahoma City, 2009.

Palazzo FF, Warner OJ, Harron M, Sadana A. Misuse of the London ambulance service: How much and why? Journal of Accident and Emergency Medicine 1998;15(6):368-370 [http://dx.doi.org/10.1136 mj.15.6.368] rating its responses. Baltimore Sun, 1 July 2008, http $/ /$ articles.baltimoresun.com/2008-07-01/news/0806300239_1 emergency-responders-lights-and-sirens-arundel-county (accessed 17 April 2013)

Snooks H, Wrigley H, George S, Thomas E, Smith H, Glasper A. Appropriateness of use of emergency ambulances. Journal of Accident and Emergency Medicine 1998;15(4):212-218. [http:// dx.doi.org/10.1136/emj.15.4.212]

6. Frank SA, de Villiers PJT. An analysis of the appropriate use of the Caledon amblance service in the Overberg: A short report. S Afr Med J 1995;85(11):1185-1186.

MacFarlane C, van Loggerenberg C, Kloeck W. International EMS systems in South Africa - past, present, and future. Resuscitation 2005:64(2):145-148. [http://dx.doi org/10.1016/).

8. Meents E, Boyles T. Emergency medical services - poor response time in the rural Eastern Cape. S Afr Med J 2010;100(12):790.

9. Health Professions Council of South Africa. Capabilities of Emergency Care Providers. Pretoria: HPCSA, 2011.

10. Palumbo L, Kubincanek J, Emerman C, Jouriles N, Cydulka R Shade B. Performance of a system to determine EMS dispatch priorities. Am J Emerg Med 1996;14(4):388-390. [http://dx.doi. org/10.1016/S0735-6757(96)90056-X]

11. Lammers RL, Roth BA, Utecht T. Comparison of ambulance dispatch protocols for nontraumatic abdominal pain. Ann Emerg Med 1995;26(5):579-589. [http://dx.doi.org/10.1016 S0196-0644(95)70008-0]

12. Snooks H, Williams S, Crouch R, Foster T, Hartley-Sharpe C, Dale J. NHS emergency response to 999 calls: Alternatives for cases that are neither life threatening nor serious. BMJ 2002;325(7359):330-333. [http://dx.doi.org/10.1136/
BMJ bmj.325.7359.330]

13. Snooks HA, Dale J, Hartley-Sharpe C, Halter M. On-scene alternatives for emergency ambulance crews attending patients who do not need to travel to the accident and emergency department: A review of the literature. Emers \begin{tabular}{l} 
Med J 2004;21(2):212-215. [http://dx.doi.org/10.1136/ \\
\hline
\end{tabular} emj.2003.005199]

14. Clawson J, Olola CH, Heward A, Scott G, Patterson B. Accuracy of emergency medical dispatchers' subjective ability to identify when higher dispatch levels are warranted over a Medical Priority Dispatch System automated protocol's recommended coding based on paramedic outcome data. Emerg Med J 2007;24(8):560563 [http://dx.doi.org/10.1136/emj.2007.047928] 